\title{
Understanding Optical Trapping Phenomena: a Simulation for Undergraduates
}

\author{
Josep Mas, Arnau Farré, Jordi Cuadros, Ignasi Juvells and Artur Carnicer
}

\begin{abstract}
Optical trapping is an attractive and multidisciplinary topic that has become the center of attention of a large number of researchers. Moreover, it is a suitable subject for advanced students that requires a knowledge of a wide range of topics. As a result, it has been incorporated into some syllabuses of both undergraduate and graduate programs. In this paper basic concepts in laser trapping theory are reviewed. To provide a better understanding of the underlying concepts for students, a Java application for simulating the behavior of a dielectric particle trapped in a highly-focused beam has been developed. The program illustrates a wide range of theoretical results and features, such as the calculation of the force exerted by a beam in the Mie and Rayleigh regimes or the calibration of the trap stiffness. Some examples that are ready to be used in the classroom or in the computer lab, are also supplied.
\end{abstract}

Index Terms

Educational technology, Electromagnetic Engineering Education, Geometrical Optics, Laser Applications, Physical Optics

This paper has been partially supported by the Agency for Management of University and Research Grants (Government of Catalonia) grant MQD2007-00042 and the Institute of Education Sciences (UB) grants REDICE06 B0601-02 and REDICE08 B0801-01.

J. Mas, A. Farré, I. Juvells and A. Carnicer are with the Applied Physics and Optics Department, Universitat de Barcelona, Barcelona, Spain e-mail: artur.carnicer@ub.edu

J. Cuadros is with the Institut Químic de Sarrià, Universitat Ramon Llull, Barcelona, Spain.

J. Mas thanks a FPU grant from the Spanish Ministry of Science and Innovation. A. Farré thanks a FI grant from the Government of Catalonia. 


\section{INTRODUCTION.}

Optical tweezers (OT) are highly-focused light beams which are able to trap and manipulate small particles (from micron to nanometer size) by taking advantage of the radiation pressure effect. Nowadays, OT systems are a basic research tool in biophysics; for instance, single-molecule DNA experiments or some cellular studies require the use of OT [1]. Particle acceleration induced by radiation pressure was first reported by Ashkin [2], who was able to trap microscopic spheres using two counter-propagating laser beams. In 1986, optical trapping with a single highly focused laser beam was achieved [3]. The trapping is achieved by momentum exchange between the beam and the trapped particle via a scattering process. The force exerted on the particle (or bead) by the light can be decomposed into a scattering component, which pushes the bead in the direction of propagation of the beam, and a gradient force that attracts the bead towards regions of higher intensity. In order to create a stable trap, the axial gradient force should match the scattering force. This can be achieved by focusing the beam using a high numerical aperture (NA) objective.

In addition to their ability to manipulate small beads, OT can also constitute an useful technique for measuring forces in the microscopic world. When an external force is applied on a trapped particle, it moves towards a new equilibrium position where the optical force compensates the external force. As the trap potential is assumed to be harmonic, it can be calibrated in a process that involves obtaining the stiffness constant. Recently, several experimental methods have been developed to measure the force exerted by a light beam [4].

Forces involved in optical trapping experiments can be predicted using the generalized LorentzMie theory (GLMT) [5]. The GLMT is a general, rigorous and rather complex approach that provides accurate results. However, when the size of the particle is much smaller than the wavelength of the beam, the so-called Rayleigh approximation can be used [6]. The Rayleigh approach is simpler than GLMT and supplies accurate enough results for most purposes within its range of applicability. In contrast, the ray optics model (Mie regime) provides a simple and intuitive description of optical trapping for particles whose diameter is larger than the wavelength of the laser used [7].

Despite the fact that OT are still a very active research area, optical trapping experiments are being introduced into some undergraduate and Master's programs in Photonics, Nanotechnology 
or Biophysics. OT currently represent an attractive and multidisciplinary area of study suitable for advanced students with a knowledge of a wide range of fields. For instance, building an OT setup involves Optical Engineering (optical system design, microscopy), Physics (electromagnetic theory, fluid mechanics), Photonics (laser technology), Electrical Engineering (signal processing, hardware control), etc. Related to this, several papers have been published pointing out the usefulness of OT as an educational tool [8] [9].

Considerable knowledge of the underlying theories is necessary to make the most of OT experiments. As the number of published papers in this field is huge, students (and instructors) will have to access a large amount of reference work. In order to provide basic and digestible information, the use of a numerical simulation as a complementary tool is suggested. Some OT simulations can be found on the Internet. For instance, the reader can look up references [10] (a simple animation of a trapped bead ) and [11] (a nice illustration of a DNA stretching experiment).

In this paper, a computer program designed to train students or non-specialists in the basic Rayleigh and Mie theories is presented; in addition, some illustrative results in OT theory that can be carried out using the same piece of software are discussed. The article is organized as follows: in section II, the basic concepts involved in optical trapping theory, including force calibration are reviewed. How the application works is described in section III and in section IV several classroom activities are introduced. Some results from laser trapping theory using the program are analyzed in section V. A preliminary assessment of the innovation is included in section VI and finally, the conclusions are presented in section VII.

\section{OPTICAL TRAPPING REVIEW.}

Ashkin [2] demonstrated that a highly focused beam is able to accelerate microscopic objects. Photons can interact with matter by transferring their momenta, which means that light is able to exert a force on a particle. Optical tweezers setups require the use of a microscope objective to concentrate the photons coming from a laser beam at a tiny spot. In this way, the target particle interacts with as many photons as possible since the momentum of each single photon is very small. A wide spectrum of accurate and sophisticated theories has gradually been developed to explain the trapping of particles of different sizes (see section VI of [4] for a detailed overview). None of them provides an exact description of an optical trap, but predictions from the numerical 
solutions of all the existing models span a significant range of experimental situations, leading to a fairly good understanding of the trapping problem. However, for spherical particles (beads), the problem is usually described by approximations, which depend on the radius of the object used in the experiment and either the wavelength or the waist size of the beam:

- Rayleigh regime $\left(a<w_{0}\right)$ : forces acting on the bead are described using electromagnetic theory $[3]^{1}$

- Mie regime $(a \gg \lambda)$ : the interaction may be calculated by means of ray optics [7].

\section{A. Forces in the Rayleigh regime}

In the Rayleigh regime, spherical particles become dipoles that scatter light. Let us suppose a bead is illuminated by a $\mathrm{TEM}_{00}$ linearly polarized (1,0) paraxial Gaussian beam $\vec{E}(x, y, z)$ [12], propagating along the $z$-axis with beam waist radius $w_{0}$ and wave-number $k=2 \pi / \lambda ; \lambda$ is the wavelength in the considered media. Let $n_{1}$ and $n_{2}$ be the refractive indices of the medium and the particle respectively; $a$ is the radius of the particle; $m=n_{2} / n_{1} ; c$ is the speed of light; $\epsilon_{0}$ is the permittivity of free space; and $\lambda_{0}=\lambda n_{1}$ is the wavelength in the vacuum. Then the beam intensity, $I(x, y, z)$, i.e., the time-average of the Poynting vector, is written as:

$$
I(x, y, z)=\frac{1}{2} n_{2} c \epsilon_{0}|\vec{E}(x, y, z)|^{2} .
$$

The polarization induced on the dipole is $\vec{p}=\alpha \vec{E}=4 \pi n_{1}^{2} \epsilon_{0} a^{3} \frac{m^{2}-1}{m^{2}+2} \vec{E}$, where $\alpha$ is the polarizability of the particle. The laser beam exerts a force $\vec{F}$ on the dipole that has two different components: gradient $\vec{F}_{g}$ and scattering $\vec{F}_{s}$. The former is proportional to the gradient of the wave intensity, which means that $\vec{F}_{g}$ points towards the focus of the microscope objective (see Figure 1). Following [6], $\vec{F}_{g}$ is written as:

$$
\vec{F}_{g}=\vec{\nabla}[\vec{p} \cdot \vec{E}]=2 \pi \frac{n_{1}}{c} a^{3} \frac{m^{2}-1}{m^{2}+2} \vec{\nabla} I(x, y, z) .
$$

\footnotetext{
${ }^{1}$ The dipole model in the Rayleigh approximation requires a homogeneous electric field over the whole particle, which leads to a restriction on the size of the particle $a<w_{0}$. The Rayleigh regime is accurate enough to describe lateral forces, which are what most experiments are concerned with. Nevertheless, as Harada et al. previously showed [6], a remarkable discrepancy appears in the axial component, which is not accurately described above $a=\lambda / 20$.
} 


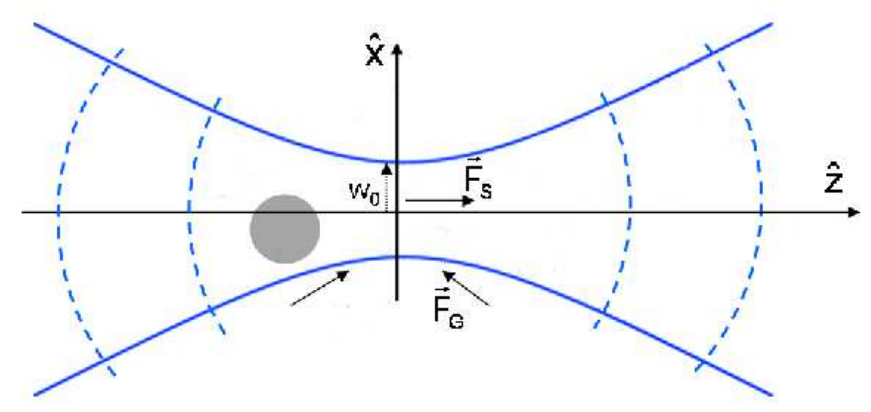

Fig. 1. Optical forces acting on a micro-sphere located near the focus of the objective lens

The second component of the force is due to the scattering, and depends on the number of photons hitting the bead. It is an axial force that pushes the sample along in the $z$-direction:

$$
\vec{F}_{s}=\frac{n_{1}}{c} \frac{8}{3} \pi k^{4} a^{6}\left[\frac{m^{2}-1}{m^{2}+2}\right]^{2} I(x, y, z) \hat{z}
$$

The total force exerted on the particle is $\vec{F}=\vec{F}_{g}+\vec{F}_{s}=F_{g}^{x} \hat{x}+F_{g}^{y} \hat{y}+\left(\left|F_{s}\right|+F_{g}^{z}\right) \hat{z}$. The two contributions to the total force, scattering and gradient, compete along the $z$-direction (optical axis). The $z$-component of the total force is zero $\left(F^{z}=\left|F_{s}\right|+F_{g}^{z}=0\right)$ for

$$
z_{\text {trap }}=\frac{3}{4 k^{4} \alpha}\left(1-\sqrt{1-\frac{4}{9} k^{10} \alpha^{2} w_{0}^{4}}\right) .
$$

At this point, not far from the focus of the Gaussian beam, the transverse components of the force are also zero and therefore the micro-sphere remains in an equilibrium position. In order to produce a stable trap, the gradient force along the optical axis has to be larger than the scattering contribution. This is true when [3]:

$$
\frac{3 \sqrt{3}}{128 \pi^{5}}\left[\frac{m^{2}-1}{m^{2}+2}\right] \frac{\lambda^{5}}{a^{3} w_{0}^{2}} \geq 1
$$

This relation is fulfilled only when the laser beam waist $w_{0}$ is small enough. For this reason OT must be designed using microscope objectives with high NA; if not, as the scattering force increases, the gradient component does not compensate the scattering force along the $z$-axis. 


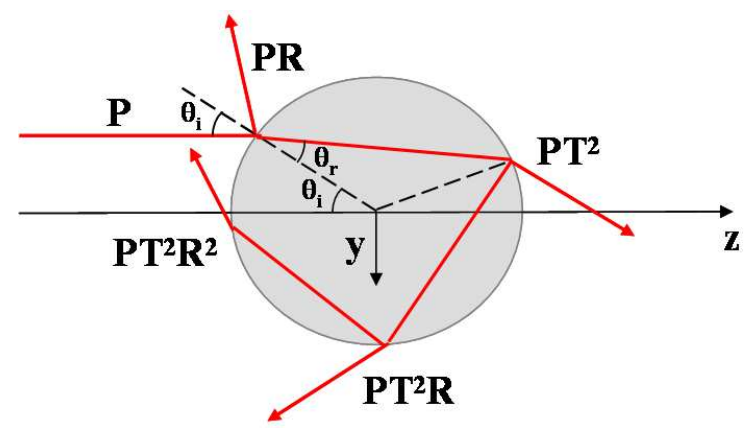

Fig. 2. Optical ray path within a spherical particle

The main drawback of the Rayleigh regime comes from the lack of accuracy in the predictions for high NA because the underlying theory has an intrinsically paraxial nature. In these cases the theory is restricted to providing a qualitative description of the system [6], [13].

\section{B. Forces in the Mie regime}

As explained above, if the diameter of the particle is much larger than the wavelength, the force exerted by the laser beam on a spherical particle is computed using the ray optics approach. When the beam interacts with the particle, rays change their direction according to the laws of geometrical optics. The interaction is described taking into account the reflections and refractions inside the particle (see Figure 2). The power $P$ of the incident ray is reduced to $P T^{2} R^{q}$ after $q$ reflections, where $R$ and $T$ stand for the Fresnel coefficients [14]. Ashkin derived analytical expressions for the transverse (gradient) and longitudinal (scattering) components of the force exerted by a single ray of power $P[7]$ :

$$
\begin{aligned}
& F_{s}=\frac{n_{1} P}{c}\left[1+R \cos \left(2 \theta_{i}\right)-T^{2} \frac{\cos \left(2 \theta_{i}-2 \theta_{r}\right)+R \cos \left(2 \theta_{i}\right)}{1+R^{2}+2 R \cos \left(2 \theta_{r}\right)}\right] \\
& F_{g}=\frac{n_{1} P}{c}\left[R \sin \left(2 \theta_{i}\right)-T^{2} \frac{\sin \left(2 \theta_{i}-2 \theta_{r}\right)+R \sin \left(2 \theta_{i}\right)}{1+R^{2}+2 R \cos \left(2 \theta_{r}\right)}\right]
\end{aligned}
$$

where $\theta_{i}$ and $\theta_{r}$ are the angles of incidence and refraction, respectively.

The ray optics approximation is particularly useful when dealing with large beads. The model is not restricted to low-convergence beams, so it not only explains the behavior of the trap, but also gives a reasonable estimate of its mechanical properties in real experiments [13]. 


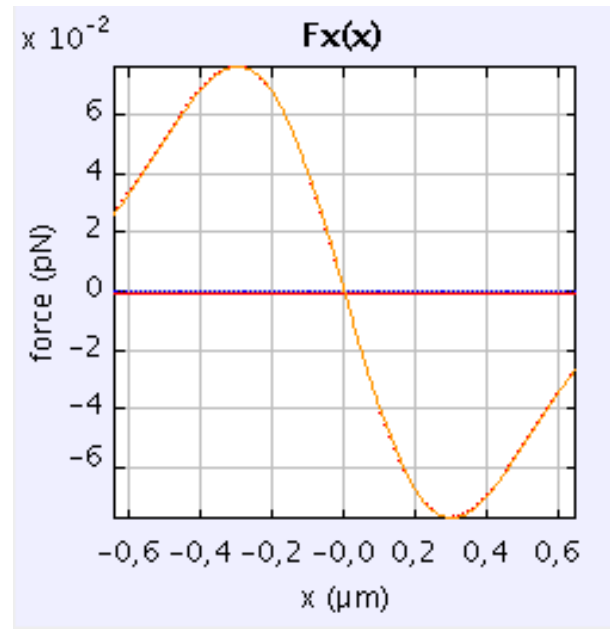

Fig. 3. Force component $F^{x}(x)\left(\lambda=1064 \mathrm{~nm}, P=100 \mathrm{~mW}, w_{0}=0.6 \mu \mathrm{m}, n_{1}=1.33, n_{2}=1.58, a=50 \mathrm{~nm}\right)$.

\section{Measurement of Transverse forces}

In both regimes, the transverse force components $F^{x}=F_{g}^{x}$ (and $F^{y}=F_{g}^{y}$ ) exerted on a particle vary linearly for small displacements from the center of the trap (Figure 3). When the bead is moving close to the center, i.e., within about half of the beam waist radius $w_{0} / 2$, the force $F^{x}$ is proportional to the position $x, F^{x}=-k_{x} x$, and therefore, the potential well $U$ is harmonic $U=\frac{1}{2} k_{x} x^{2}$. Similarly, the force in the $z$-direction can also be described by $F^{z}=-k_{z} z$.

Since the force exerted by the laser beam is proportional to the displacement of the sample, OT are used to measure forces in the microscopic world. As the spring constant $k_{x}$ can be obtained experimentally, a precise measurement of the position of the sample within the laser spot is required to obtain the force exerted by the beam. The procedure for obtaining $k_{x}$ is based on the analysis of the Brownian motion of the trapped sample.

One calibration method for $k_{x}$ is based on the reconstruction of the optical potential $U$ from the histogram of the position of the bead. The particle moves randomly within the trap, but the bead spends more time in those places where the optical potential energy is lower. According to Florin [15], the probability distribution of the position $x$ is related to the potential energy thus:

$$
p(x) \propto \exp \left(-\frac{U}{k_{B} T}\right)=\exp \left(-\frac{k_{x} x^{2}}{2 k_{B} T}\right)
$$

where $k_{B}$ is Boltzmann's constant and $T$ is the temperature. For a large number of measurements of $x$, the histogram provides a good estimate of the spatial probability density $p(x)$ (except for 
a normalization constant) and the trap stiffness $k_{x}$ can be estimated form the variance $\sigma_{x}^{2}$ of $x$

$$
k_{x}=\frac{k_{B} T}{\sigma_{x}^{2}}
$$

The motion of a micro-particle can be easily simulated using Newton's second law (Langevin equation)

$$
m \ddot{x}=-\gamma \dot{x}-k_{x} x+\sqrt{2 k_{B} T \gamma} \xi(t)
$$

Here, $-\gamma \dot{x}$ is the drag force, $\gamma=6 \pi \eta a$ is the drag coefficient, $\eta$ is the viscosity of the medium and finally, $\sqrt{2 k_{B} T \gamma} \xi(t)$ is a random force that accounts for the Brownian motion due to the water molecules. For polystyrene or silica beads or cells, the Reynolds number is very small and therefore the inertial term $m \ddot{x}$ in equation 9 can be ignored [16].

\section{DESCRIPTION OF THE APPLICATION}

The applet simulates the behavior of a spherical dielectric particle trapped by means of a laser beam focused by a microscope objective. The program and the source code can be downloaded from http://code.google.com/p/optical-tweezers/. The simulation, including the movement of the

particle, is confined to the $x z$-plane. The calculation of the trapping force is carried out using the geometrical optics approach (Mie approximation) or in the Rayleigh approximation, according to the size of the particle. The user can select the proper working conditions via the selector located in the left-hand panel.

In the left-hand panel (Figure 4), a transverse section of the optical trap is shown. The $z$-axis corresponds to the beam propagation direction while the $x$-axis is the transverse coordinate. Notice that light comes from the bottom of the panel. Both the particle studied and the trapping light beam are represented in this panel. The optional buttons below the trap diagram allow the visualization of three vectors representing the scattering, gradient and net force exerted by the beam at the current position of the particle. The current modulus of the net force is shown below the trap diagram.

The user can click on the particle and drag it to a new position. Starting from the position selected, a simulation of the particle's trajectory is calculated when the "Animation" button is clicked. For every frame of animation, the time is shown below the diagram. The button "Take bead to focus" is used to place the particle in the center of the panel. The data required to perform this simulation come from the dynamic solution of the Langevin equation (Equation 9). 


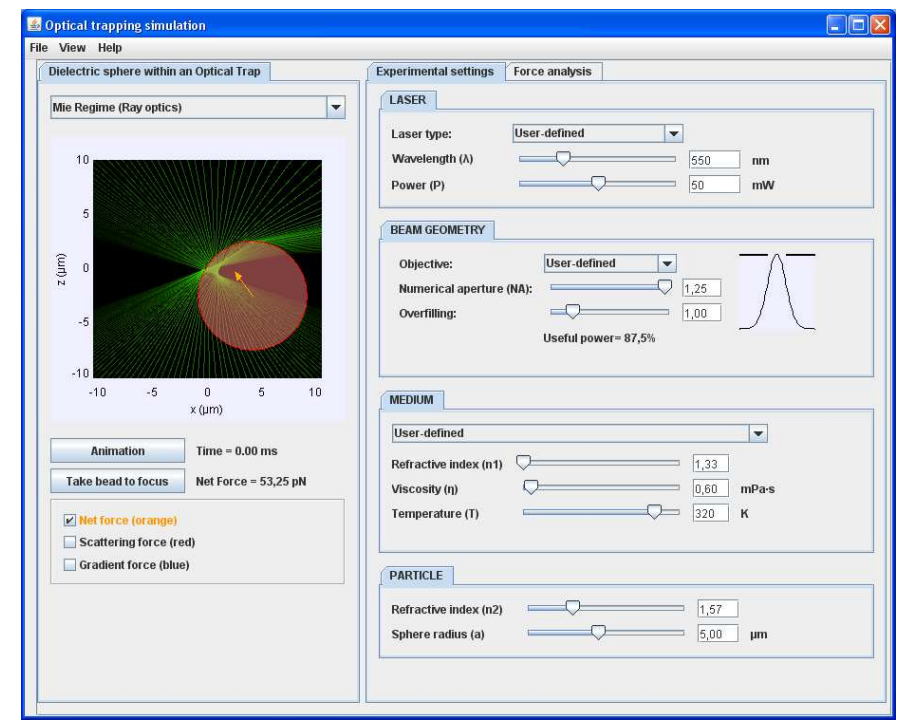

Fig. 4. Experimental settings tab

The time step used for the integration of the differential equation can be modified by activating the optional "Time step" slider in the "View" menu. Special care must be taken when selecting large time-step values; if the time step is much longer than the characteristic timescale of the movement of the bead within the optical trap (which depends on the experimental settings selected), the program could return a non-realistic animation.

In the Mie regime the light beam is represented as a group of several converging light rays in the $x z$-plane, which are refracted through the dielectric particle. The momentum transferred to the particle, i.e., the force exerted by the beam, is obtained by using Equation 2 and adding the contribution of each ray. The power associated with each ray is weighted according to a Gaussian intensity profile. In the Rayleigh regime, the laser beam is represented by the intensity map of the Gaussian beam.

In the right-hand panel there are two different tabs for configuring and analyzing the experiment. In the first tab ("Experimental settings") the user can tune the values of the variables. The parameters available are the power and the wavelength of the laser, the NA of the microscope objective, the overfilling factor, the radius and refractive index of the trapped particle, the viscosity and refractive index of the medium, and the temperature. Notice that when using high NA objectives, the divergence of the beam cannot be easily related to the beam waist. In 


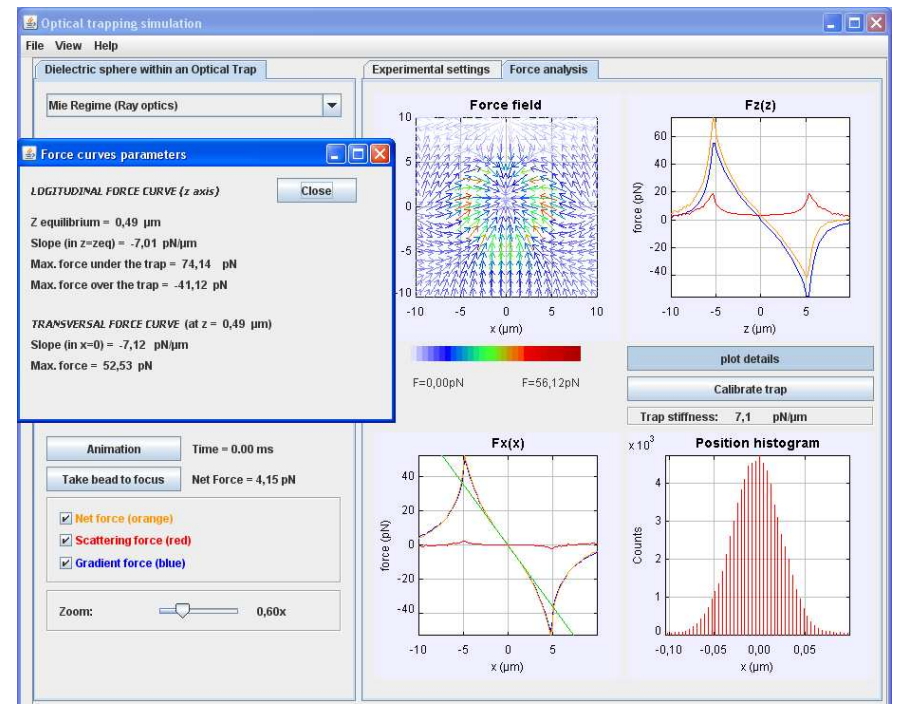

Fig. 5. Force analysis and calibration tab

this case, when the paraxial formula is not strictly valid, the values of NA in the Beam Geometry tab are displayed in red. This is noticeable when working in the Rayleigh approximation.

The overfilling factor $\gamma$ is the ratio of the full width at half maximum (FWHM) of the beam intensity profile, to the diameter of the objective entrance pupil. Modification of the overfilling value changes the fraction of power that crosses the entrance pupil, but also affects the shape of the force field and the stability of the trap, as analyzed in the section V. In the program the overfilling factor setting is only available for the ray optics regime, where the relative power associated with each ray changes according to the overfilling factor. In the Rayleigh regime, the overfilling setting is not available because the beam is no longer Gaussian and the computational cost would be too high to perform the calculations in real time during the animation.

By clicking on the "Force analysis" tab the user can visualize the force field created by the optical trap. In particular, the behavior of the transverse $F^{x}(x)$ and longitudinal $F^{z}(z)$ components of the force is shown. The scattering and gradient components and the net force can be selected independently by clicking on the optional buttons on the left panel. The transverse force curve is calculated at $z_{\text {trap }}$ (but if $z_{\text {trap }}$ does not exist, $F^{x}(x)$ is obtained at $z=0$ ). By clicking the "Plot details" button, a new window pops up that indicates a set of parameters obtained from the force curves. If $z_{\text {trap }}$ can be found, the equilibrium position, the maximum values of the force and the slope of the curves are calculated (see Figure 5). Notice that $z_{\text {trap }}$ 
defines the position where the $z$ component of the total force is zero. Despite the fact this is a necessary condition for trapping, the existence of $z_{\text {trap }}$ does not assure a stable trap.

If the configured beam is able to trap particles, the net transverse force component $F^{x}(x)$ displays linear behavior in the central area of the trap. Its slope provides a measure of the stiffness $k_{x}$ of the trap, as explained in the previous section. When the "Calibrate trap" button is clicked, the transverse stiffness of the trap is obtained by means of the calibration method based on the analysis of the Brownian motion. While the projection of the trajectory of the bead on the $x$-axis is calculated, the $x$-position histogram is updated and the trap stiffness is continuously calculated from the $x$-standard deviation and shown below the histogram. This procedure emulates a continuous position measurement in the laboratory. When the number of points collected is high enough and if the stiffness value stabilizes, the histogram displays a characteristic Gaussian shape. The stiffness obtained with this method should match the slope of the transverse force curve.

\section{Suggested EXERCISES}

In this section, some classroom exercises to be carried out using the applet are presented.

\section{A. Ray optics regime}

1) Choose the Mie regime approach and select the visualization of the scattering and gradient components and the net force. Set the experimental variables with the following realistic values:

- Laser: A 50 mW, Nd:YVO4 doubled laser beam @ $\lambda=532 \mathrm{~nm}$.

- Beam geometry: An oil-immersion objective 100x with NA $=1.25$ and $\gamma=1$.

- Medium: Water $n_{1}=1.33$, temperature $T=300 \mathrm{~K}$, viscosity $\eta=0.85 \mathrm{mPa}$.

- Particle: polystyrene spheres; radius $a=2.5 \mu \mathrm{m}$ and refractive index $n_{2}=1.58$.

2) Click on the optional force buttons (left panel). By pressing the "Animation" button, the bead is displaced to the equilibrium position if stable trapping is possible. Beam forces try to compensate the effect of the temperature (Brownian motion) to keep the particle in the trap. The "Force analysis" tab shows the force fields. The equilibrium position $z_{\text {trap }}$ is slightly shifted to the upper side of the diagram from the focusing plane $(z=0)$. This effect is corroborated by the $F^{z}(z)$ diagram, as the net force becomes zero in $z_{\text {trap }} \neq 0$. After 
stopping the simulation, the bead can be centered in the focusing plane by clicking on the "Take bead to focus" button. Click on the "Calibrate trap" button to run the Brownian motion simulation. Verify that the stiffness reaches a stable value when the histogram shape becomes Gaussian. When the simulation is stopped, the stiffness value obtained provides an adjustment to the slope of $F^{x}$.

3) When the simulation has finished, the user can click on the bead and drag it to an arbitrary position in the panel. The user can verify that if the bead is located near the focus, trapping is possible (notice the directions of force vectors).

4) Now set the refractive index of the particle to $n_{2}=1.2$. Is the beam able to trap the bead?

5) Repeat the analysis using different values for NA, power, overfilling or particle size. Discuss under what conditions is it easiest to trap the particle?

- high/low NA values?

- high/low laser power?

- high/low overfilling values?

- large/small particle size?

\section{B. Rayleigh regime}

Now, a partial repetition of the previous exercise is proposed. Initially, the variables are set to the following values:

- Laser: A 50 mW, Nd:YVO4 doubled laser beam @ $\lambda=532$ nm.

- Beam geometry: beam waist $w_{0}=0.3 \mu \mathrm{m}$.

- Medium: Water $n_{1}=1.33$, temperature: $T=300 \mathrm{~K}$, viscosity $\eta=0.85 \mathrm{mPa}$.

- Particle: polystyrene spheres; radius $a=30 \mathrm{~nm}$ and refractive index $n_{2}=1.58$.

Is stable trapping possible in such conditions? Discuss what must be changed to improve the performance of the system.

\section{Optical tweezers design.}

Now, the target is to design a system that can trap polystyrene spheres in water at $\mathrm{T}=298 \mathrm{~K}$. The set-up is illuminated with a $\lambda=532 \mathrm{~nm}$ laser. Taking into account different sizes of the beads:

1) Discuss which available objective is more convenient, and what is the amount of overfilling and the minimum power required to create a stable trap. 


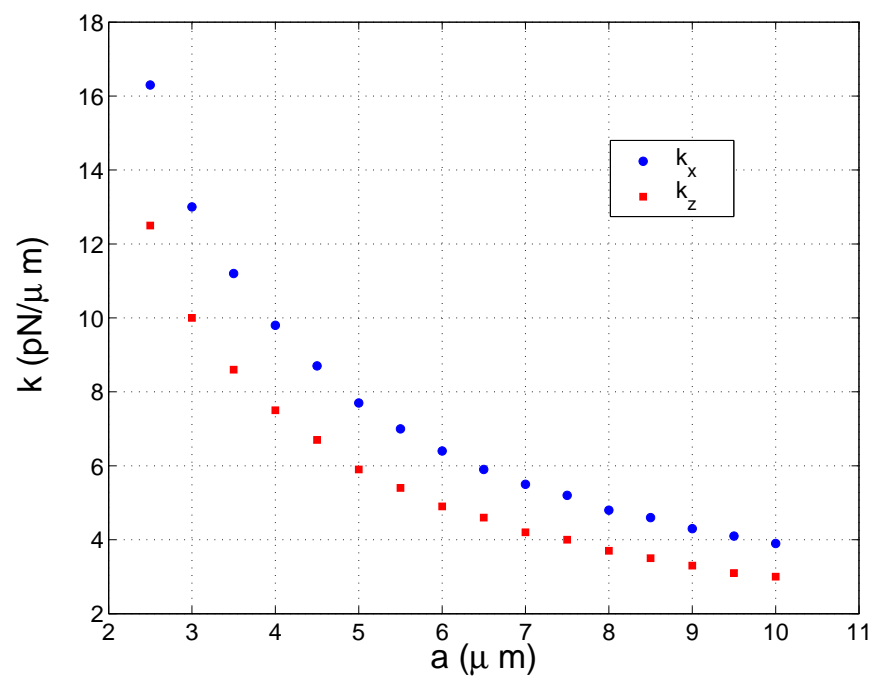

Fig. 6. Lateral and axial trap stiffnesses for different bead sizes in the ray optics regime. Values used in this simulation: $\lambda_{0}=$ $532 \mathrm{~nm}, P=50 \mathrm{~mW}, \mathrm{NA}=1.2, \gamma=1, n_{1}=1.33, \eta=0.6 \mathrm{mPa} \mathrm{s}, T=320 \mathrm{~K}$, and $n_{2}=1.57$.

2) What are the stiffness and the trapping position $z_{\text {trap }}$ ?

\section{VERIFICATION OF SOME RESULTS FROM OPTICAL TRAPPING USING THE APPLET}

The software enables a comprehensive and interactive analysis of the interplay between the experimental parameters describing the laser-sample interaction. Simple dependencies of the trap stiffness $k_{x}$ on, for instance, the laser power can be easily simulated; nonetheless, it is the study of more complicated behavior, which cannot be readily inferred from a mathematical model, that turns the program into a useful tool. In this section, a set of tasks to analyze the key parameters of the two models described in section II are introduced. This should ultimately lead to a better and deeper understanding of the properties of optical traps.

The use of numerical analysis becomes particularly important in the ray optics limit. In this context, the vast majority of the questions about the trap features can be uniquely solved through a computer simulation.

1) Simulate the values of the lateral and axial trap stiffnesses for different bead sizes. Notice that, as Figure 6 shows, both curves should exhibit the same $1 / a$ decrease as that found in experiments [17]. Because the maximum force applied by the beam occurs at the edge of the sphere, its value will not depend on the size of the sample [7]. 


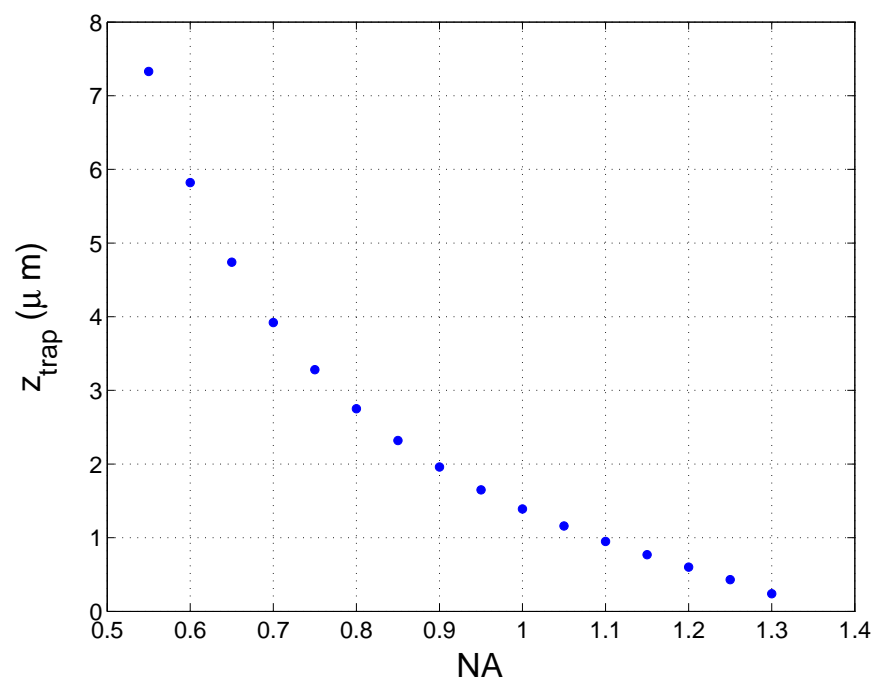

Fig. 7. The axial trapping position as a function of the objective's numerical aperture. Values used: $\lambda_{0}=1064 \mathrm{~nm}, P=50$ $\mathrm{mW}, \gamma=1, n_{1}=1.33, \eta=0.6 \mathrm{mPa} \mathrm{s}, T=320 \mathrm{~K}$, and $n_{2}=1.57$, and $a=5 \mu \mathrm{m}$.

2) Analyze the relation between the equilibrium position of a trapped particle and the NA of the objective lens. As NA decreases the micro-sphere is displaced forward in the $z$ direction, as shown in the example (Figure 7). The increase in the scattering force for low NA is the reason behind this change. Photon scattering moves the equilibrium position of the sample away from the focus until this force can no longer be balanced by the restoring gradient component; at NA $\leq 0.5$, in this case. This explains why the use of such a high NA objectives is required to create stable traps. A similar behavior is obtained in the Rayleigh regime.

3) Study the maximum trapping force, as dependent on the overfilling $\gamma$. As the overfilling increases, the axial force should also increase because of the diminishing scattering effect (Figure 8). Beyond a certain optimum overfilling, $\gamma \sim 1.1$ in the example, close to the values suggested by experiments $(\gamma=1$ and $\gamma=0.91$ in [4], [18]), the lateral component will start limiting the escape force, that is, the force needed to pull the sample out of the trap in any direction. The value of $\gamma$ at this intersection point is considered to provide the best performance of the trap, corresponding to the case when the objective is slightly overfilled by the beam.

In the Rayleigh approximation the force is calculated through a couple of analytical expression 


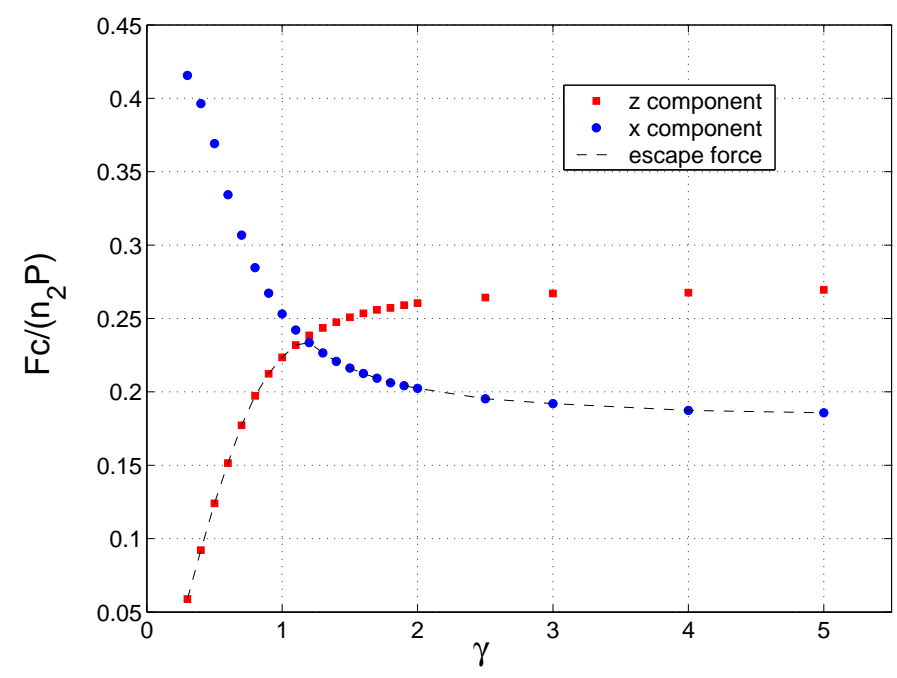

Fig. 8. The normalized maximum force depending on the degree of overfilling along the axial and transversal directions. The dashed line indicates the escape force as defined in the text. Values used: $\lambda_{0}=1064 \mathrm{~nm}, P=50 \mathrm{~mW}, \mathrm{NA}=1.2, n_{1}=1.33, \eta$ $=0.6 \mathrm{mPa} \mathrm{s}, T=320 \mathrm{~K}$, and $n_{2}=1.57$, and $a=5 \mu \mathrm{m}$.

considering all the variables involved except the overfilling $\gamma$ (see equations 2 and 3). This makes the analysis of this model more accessible. However, if one computes the force in the trapping plane $z_{\text {trap }}$, the trap stiffness no longer has a trivial relation with the parameters because of the dependence of the beam width $w$ on the axial position $z$. Taking this effect into account in the experiments is important because it can yield a change in $k_{x}$ by a factor of 1.5 (with respect to the stiffness at the $z=0$ plane) [4]. However, the correction is negligible for particles with $a<\lambda / 20$.

1) In this regime, the study of the relationship between $k_{x}$ and the refractive index of the sample is especially interesting. Set the refractive index of the medium $n_{1}$ to that of water, 1.33, or similar, and choose a value for $n_{2}$ close to this number. Figure 9 shows the behavior of $k_{x}$ as a function of $n_{2}$.

The result graphically explains why the trapping of particles with a refractive index lower than that of the surrounding medium $\left(n_{2}<n_{1}\right)$ is unfeasible. Likewise, it explains the higher lateral forces that can be achieved in the laboratory using polystyrene beads $\left(n_{2}=1.57\right)$ compared to those achieved using silica particles $\left(n_{2}=1.45\right)$. In practice, the refractive index of the microparticles used in the experiments cannot be increased 


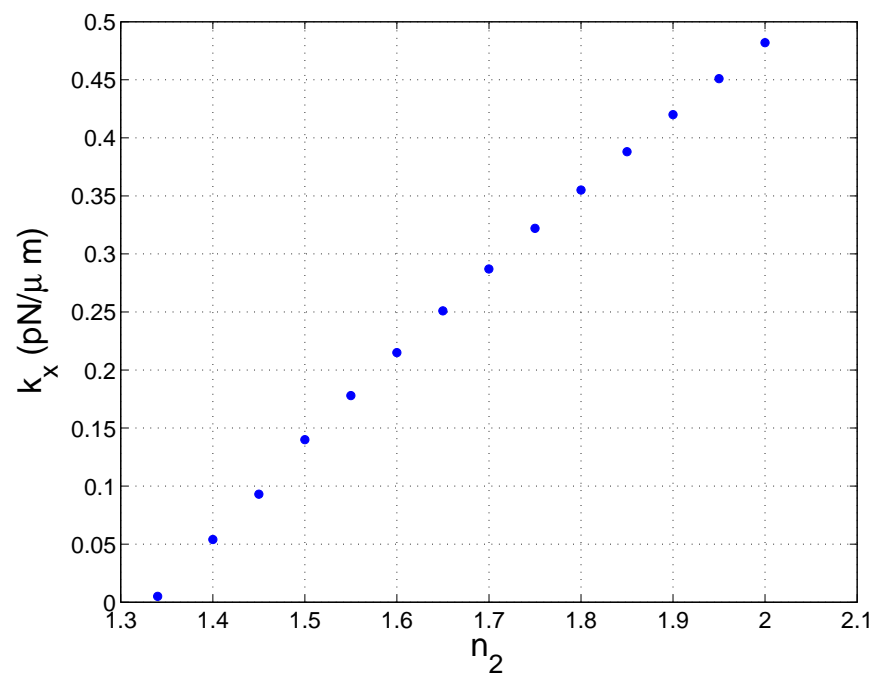

Fig. 9. Relation between $k_{x}$ and the refractive index of the particle. Values used: $\lambda_{0}=1064 \mathrm{~nm}, P=50 \mathrm{~mW}, w_{0}=0.52 \mu \mathrm{m}$, $n_{1}=1.33, \eta=0.6 \mathrm{mPa} \mathrm{s}, T=320 \mathrm{~K}$, and $a=40 \mathrm{~nm}$.

indefinitely because the trap becomes unstable [19].

A similar variation in $k_{x}$ is found in the ray optics regime. The reflection and transmission coefficients introduce the dependence on $n$.

2) Verify that the trap stiffness $k_{x}$ depends on $a$ following a cubic function (see Figure 10 and Equation 2). The combination of this result with the $1 / a$ behavior for big particles gives rise to an absolute maximum of $k_{x}$ for diameters of $1-2 \mu \mathrm{m}$ [17], which are the bead sizes typically chosen in the laboratory.

\section{ASSESSMENT OF THE INNOVATION}

The applet presented in this paper was used by 18 students as part of a Photonics course in the first semester of the 2009-2010 academic year. Photonics is an elective course for senior undergraduates in Physics at the Universitat de Barcelona. This tool was the main object of a 2-hour computer lab session on OT which used an adapted version of the first suggested exercise (see section IV-A). The topic was previously presented in a lecture class. About a month after having used the program, an assessment questionnaire was handed out to the students. This assessment instrument was designed to include two different measurements: the first half of the questionnaire deals with students' perception of the applet, the second part is a short learning 


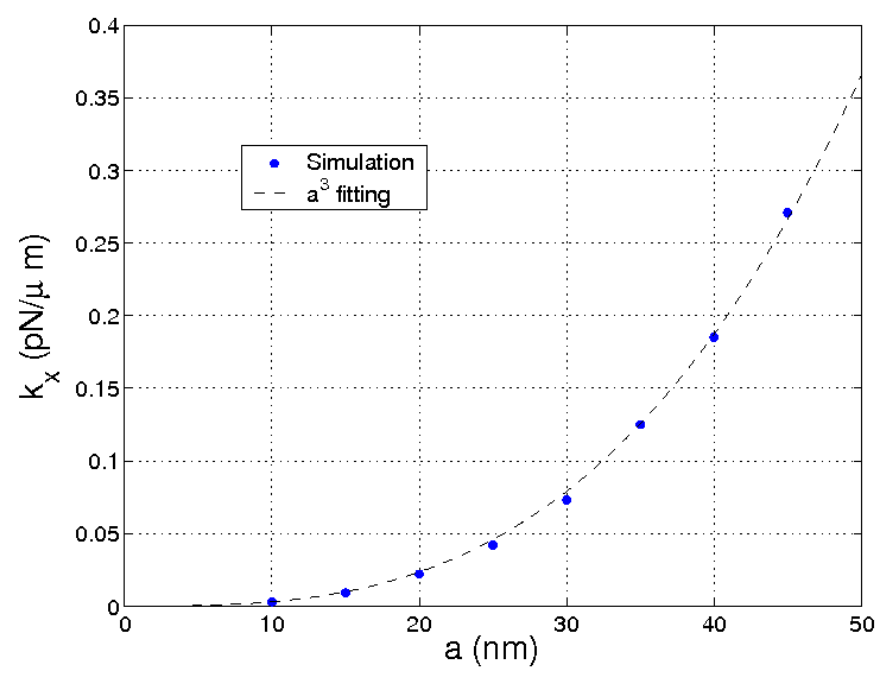

Fig. 10. Trap stiffness for different values of a, in the Rayleigh regime. Values used: $\lambda_{0}=1064 \mathrm{~nm}, P=50 \mathrm{~mW}, w_{0}=0.52$ $\mu \mathrm{m}, n_{1}=1.33, \eta=0.6 \mathrm{mPa} \mathrm{s}, T=320 \mathrm{~K}$, and $n_{2}=1.57$.

retention test. All 18 students turned in the questionnaire and all 18 responses were analyzed.

\section{Students' perception}

The perception part of the questionnaire included a 4-item Likert scale and two open-ended questions. The Likert scale was meant to measure the students' perception on the usefulness of the applet for learning. The four 4-level items of the scale are shown in Table I. The following two open-ended asked for the best (or most remarkable) and the worst (or most improbable) aspects of the applet. The results for each item of the Likert scale are presented in Table I. They show a highly positive opinion of the students on the applet; rescaling to a $0-100$ scale, the global average sums up to 83 .

In the open-ended questions, students mention the following ideas:

- As for the positive aspects, they remark that the program helps learning (11 answers), especially improving visualization (9) and understanding (2) of the phenomena, and that it is user-friendly and intuitive (5).

- As for the negative side, or the characteristics that could be improved, users highlight that they would need more time and explanations for using the applet in the lab session (6) and some interface related issues (5) specifically that graphical resolution of some charts is too 
TABLE I

FREQUENCIES FOR THE RESPONSES TO THE LIKERT SCALE ITEMS OF THE QUESTIONNAIRE. QUESTIONS AND ANSWERS WERE WRITTEN IN CATALAN IN THE QUESTIONNAIRE AND HAVE BEEN TRANSLATED FOR THIS PAPER. GRAYED CELL HIGHLIGHT THE MODE OF THE ANSWERS TO EACH QUESTION.

\begin{tabular}{|c|c|c|c|c|c|}
\hline Question & 1 (Disagree) & 2 (Tend to disagree) & 3 (Tend to agree) & 4 (Agree) & N/A \\
\hline $\begin{array}{l}\text { I believe that the applet has favored my } \\
\text { learning of the course subject. }\end{array}$ & 0 & 1 & 10 & 6 & 1 \\
\hline $\begin{array}{l}\text { I think the applet make easier to learn } \\
\text { how optical tweezers work. }\end{array}$ & 0 & 1 & 5 & 12 & 0 \\
\hline $\begin{array}{l}\text { I would recommend the applet to } \\
\text { be used in following editions of the } \\
\text { course. }\end{array}$ & 0 & 1 & 5 & 12 & 0 \\
\hline $\begin{array}{l}\text { The experience done with the applet } \\
\text { has allowed me to better understand } \\
\text { the different variables that interplay in } \\
\text { optical tweezers. }\end{array}$ & 0 & 3 & 3 & 10 & 2 \\
\hline
\end{tabular}

low, that the application is not intuitive enough and that it has a slow response; five students did not report any negative aspect.

\section{Learning retention}

The second part of the questionnaire included two open-ended questions to assess learning retention. Before discussing the results in this part, it is important to highlight that the assessment wasn't announced previously and that no further work on this topic was done between the academic use of the applet and the assessment data collection (i.e. the lab reports weren't collected yet and there were no exams on this topic in this period).

The two open-ended questions that were asked read as follows:

1) Write down 3 variables that affect the stiffness of optical tweezers in the Mie regime.

2) Explain qualitatively how the trap stiffness changes the amplitude of the Brownian motion of a trapped particle.

The first question was left blank by 6 students, 8 students gave two correct variables and 4 wrote down a fully correct answer. Thus, $67 \%$ of the students gave an at least partially correct answer. The second question was answered by 11 students and all of them gave a correct response. 
Thus, $61 \%$ of the students got it right. Both percentages show that a significant learning took place while using the applet and that part of it remains a month after the learning activity. Both set of data, students' perception and learning retention show consistently that the presented innovation is useful for learning this highly complex topic.

\section{CONCLUDING REMARKS}

Building an OT set-up from scratch that is able to measure forces is a wonderful laboratory project for advanced undergraduate students. The task requires considerable knowledge of the various physical theories involved and also some practical skills. Digital simulations can be a powerful training tools if they are designed to fill the gap between theory and practice. In this paper, a comprehensive Java applet that covers relevant aspects of optical trapping theory has been presented. In addition, some exercises related to real experimental situations have been proposed. The use of the applet in the stage prior to designing the system enables students to become more confident in the lab since they acquire sound theoretical knowledge with minimum effort. A preliminary assessment, analyzing students' perception and learning retention data, suggests it can be a helpful tool to improve learning on this topic.

\section{APPENDIX}

\section{ABOUT THE SOFTWARE}

The complete source code and the executable file can be downloaded from http://code.google. com/p/optical-tweezers/. The code for this software is written in Java implementing classes from the Open Source Physics Library (http://www.compadre.org/OSP/) and the Apache Commons Mathematics Library (http://commons.apache.org/math/). The program was compiled using the Sun Java SDK version 1.6.0_03. The software is licensed under a GNU General Public License v3.

\section{ACKNOWLEDGMENT}

The authors would like to thank Dr. Mario Montes-Usategui and Dr. Xavier de Pedro for their helpful comments and suggestions. 


\section{REFERENCES}

[1] M. J. Lang and S. M. Block, "Resource letter: Lbot-1: Laser-based optical tweezers," Am. J. Phys., vol. 71, no. 3, pp. 201-215, 2003.

[2] A. Ashkin, “Acceleration and trapping of particles by radiation pressure," Phys. Rev. Lett., vol. 24, pp. 156-159, 1970.

[3] A. Ashkin, J. Dziedzic, J. E. Bjorkholm, and S. Chu, "Observation of a single-beam gradient force optical trap for dielectric particles," Opt. Lett., vol. 11, pp. 288-290, 1986.

[4] K. C. Neuman and S. M. Block, "Optical trapping," Rev. Sci. Instrum., vol. 75, no. 9, pp. 2787-2809, 2004.

[5] G. Gouesbet, "Generalized Lorentz-Mie theory and applications," Part. Part. Syst. Charact., vol. 11, pp. 22-34, 1994.

[6] Y. Harada and T. Asakura, "Radiation forces on a dielectric sphere in the rayleigh scattering regime," Opt. Commun., vol. 124, pp. 529-541, 1996.

[7] A. Ashkin, "Forces of a single-beam gradient laser trap on a dielectric sphere in the ray optics regime," Biophys. J., vol. 61, pp. 569-582, 1992.

[8] S. P. Smith, S. R. Bhalotra, A. L. Brody, B. L. Brown, E. K. Boyda, and M. Prentiss, "Inexpensive optical tweezers for undergraduate laboratories," Am. J. Phys, vol. 67, no. 1, pp. 26-35, 1999.

[9] R. Pastel, A. Struthers, R. Ringle, J. Rogers, C. Rohde, and P. Geiser, "Laser trapping of microscopic particles for undergraduate experiments," Am. J. Phys., vol. 68, no. 11, pp. 993-1001, 2000.

[10] R. DiLeonardo, “The trap forces applet," 2005, accessed 19-November-2009. [Online]. Available: http://glass.phys. uniroma1.it/dileonardo/Applet.php?applet=TrapForcesApplet

[11] "The Physics Education Technology Project: Optical tweezers and applications," 2008, accessed 19-November-2009. [Online]. Available: http://phet.colorado.edu/simulations/sims.php?sim=Optical_Tweezers_and_Applications

[12] B. Saleh and M. Teich, Fundamentals of Photonics. Hoboken, NJ: John Wiley and Sons, 2007.

[13] W. H. Wright, G. J. Sonek, and M. W. Berns, "Radiation trapping forces on microspheres with optical tweezers," Appl. Phys. Lett., vol. 63, no. 6, pp. 715-717, 1993.

[14] E. Hecht, Optics. San Francisco, CA: Adisson-Wesley, 2001.

[15] E. L. Florin, A. Pralle, E. H. K. Stelzer, and J. K. H. Hörber, "Photonic force microscope calibration by thermal noise analysis," Appl. Phys A, vol. 66, pp. S75-S78, 1998.

[16] K. Berg-Sorensen and H. Flyvbjerg, "Power spectrum analysis for optical tweezers," Rev. Sci. Instr., vol. 75, pp. 594-612, 2004.

[17] R. M. Simmons, J. T. Finer, S. Chu, and J. A. Spudich, "Quantitative measurements of force and displacement using an optical trap," Biophys. J., vol. 70, no. 4, pp. 1813-1822, 1996.

[18] L. P. Ghislain, N. A. Switz, and W. W. Webb, "Measurement of small forces using an optical trap," Rev. Sci. Instrum., vol. 65, no. 9, pp. 2762-2768, 1994.

[19] A. Van der Horst, P. D. J. Van Oostrum, A. Moroz, and A. Van Blaaderen, "High trapping forces for high-refractive index particles trapped in dynamic arrays of counterpropagating optical tweezers," Appl. Opt., vol. 47, pp. 3196-3202, 2008.

Josep Mas (Vic, 1985) studied physics at the Universitat de Barcelona (UB) and obtained the MSc in photonics degree in 2008 from the Universitat Politècnica de Catalunya. 
Currently, he is a PhD student at the Applied Physics and Optics Department of the UB and is working on holographic optical tweezers and video particle tracking for biophysical experiments.

Arnau Farré (Puigpunyent, Balearic Islands, 1982) was awarded his MSc in biophysics by the UB in September 2008 and is currently a PhD student in the field of optical tweezers and biological applications.

He is a member of the Applied Physics and Optics Department at the UB since 2006.

Mr. Farré is member of the Catalan Physical Society.

Jordi Cuadros (Barcelona, 1973) was awarded both his BSc degree (1997) and his PhD (2003) in chemistry by the Universitat Ramon Llull (URL), Barcelona.

He spent then 2 years in postdoctoral stage in the Department of Chemistry of Carnegie Mellon University, Pittsburgh (PA). Since his thesis, his research has a main focus in the use of simulations for teaching and learning physical sciences at the university level. Currently, he is an Associate Professor at the Applied Statistics Department, Institut Químic de Sarrià (URL).

Prof. Cuadros is member of the Catalan Chemical Society, the American Chemical Society and its Division of Chemical Education, and the European Science Education Research Association.

Ignasi Juvells (Manresa, 1949) received his BSc degree in physics in 1972 and his $\mathrm{PhD}$ in 1977 from the UB.

He joined the faculty of the UB in 1972, where currently, he is Full Professor and the Department Head of the Applied Physics and Optics Department. Dr. Juvells has worked on digital and optical image processing, image quality analysis, holography, optical information processing and biological and medical image applications. He teaches courses on optics and image processing in the aforementioned university.

Prof. Juvells is a member of the Catalan Physical Society, the Spanish Optical Society and the European Optical Society.

Artur Carnicer (Barcelona, 1965) received his BSc and PhD degrees in physics in 1989 and 1993 from the UB.

Currently, he is Associate Professor in the Applied Physics and Optics Department at the UB where he teaches undergraduate and graduate courses in physical optics and photonics. His research focuses on the development of computational methods for the study of highly focused electromagnetic fields.

Prof. Carnicer is member of the Catalan Physical Society and the Royal Spanish Physical Society 